\title{
Exploration analytique des intoxications par les pesticides
}

\section{Analytical investigation in pesticide intoxication cases}

\section{Éric LACASSIE*(1), Pierre MARQUET ${ }^{(1)}$, Jean-Michel GAULIER ${ }^{(1)}$, Marie-Françoise DREYFUSS ${ }^{(2)}$, Gérard LACHÂTRE ${ }^{(1,2,3)}$}

(1) Service de Pharmacologie et Toxicologie, CHU Dupuytren - LIMOGES - France (2) Laboratoire de Chimie Analytique, Université de Pharmacie - LIMOGES - France (3) Laboratoire de Toxicologie, Université de Pharmacie - LIMOGES - France

*Auteur à qui adresser la correspondance : Éric LACASSIE, Service de Pharmacologie et Toxicologie, CHU Dupuytren, 2, avenue Martin Luther-King - 87042 LIMOGES Cedex - France Tél : 0555056140 -Fax : 0555056162 - e-mail : lacassie@unilim.fr

\section{$R \hat{E} S U M E ́$}

Du fait du nombre croissant d'intoxications par les pesticides, il est important de développer des méthodes performantes permettant l'identification et le dosage des molécules appartenant à l'ensemble des classes de pesticides. Dans ce but, nous avons développé une méthode de dosage originale et sensible de 61 pesticides dans les matrices biologiques. Cette méthode utilise une procédure d'extraction solide/liquide sur support polymérique (HLB and MCX) OASIS $^{\circledR}$. Le couplage chromatographie en phase gazeuse/spectrométrie de masse (GC/MS) est utilisé pour les pesticides volatils (organophosphorés, organochlorés, phtalimides, uraciles) et un couplage chromatographie en phase liquide/spectrométrie de masse (LC/MS) pour les pesticides polaires et thermolabiles (carbamates, benzimidazoles). L'acquisition est réalisée en mode fragmentométrique (SIM). Les rendements d'extraction varient selon la nature des pesticides dosés, mais restent satisfaisants pour l'ensemble des pesticides. Les limites de détection (LOD) et les limites de

\section{SUMMARY}

Considering the huge use of pesticides on a worldwide basis, pesticides account for a small but increasing number of human acute and severe intoxication. However, intoxication cases attributed to pesticides are not always well diagnosed nor documented. In clinical and forensic toxicology, identifcation and quantification of the toxicants involved are essential for a good diagnosis. Hence, we developed an original and sensitive multiresidue methods for the detection and quantitation, in human biological matrices, of sixty one pesticides of toxicological significance in human. These methods involved rapid solid-phase extraction using new polymeric support (HLB and MCX) OASIS ${ }^{\otimes}$ cartridges. Gas chromatography-mass spectrometry (GC/MS) was used for volatile (organophosphate, organochlorine, phtalimide, uracil) pesticides and liquid chromatography-ionspray ${ }^{\circledR}$-mass spectrometry (LC/MS) for thermolabile and polar pesticides (carbamates, benzimidazoles). Acquisition was performed in the selected ion monitoring (SIM) mode. Extraction recovery varied owing to the nature of pesticides but was satisfac- 
quantification (LOQ) sont réparties entre 2,5 et $20 \mathrm{ng} / \mathrm{ml}$ et de 5 à $50 \mathrm{ng} / \mathrm{ml}$. La linéarité a été étudiée entre les différentes $L O Q$ et $1000 \mathrm{ng} / \mathrm{ml}$ pour tous les pesticides étudiés. Les résultats sont reproductibles et répétables, avec une bonne précision et une bonne justesse. Des exemples d'intoxication permettent de montrer l'intérêt diagnostique de ces méthodes : deux cas d'intoxication mortelle à l'endosulfan et au carbofuran; trois autres d'intoxication aiguë au parathion-éthyle, à la bifenthrine et à l'aldicarbe.

\section{MOTS-CLÉS}

Pesticides, GC/MS, LC/MS, SPE, sérum, toxicologie clinique et médico-légale.

\section{Introduction}

Les pesticides sont largement utilisés de par le monde en agriculture, pour la protection des cultures, mais aussi chez les particuliers. La plupart des intoxications aiguës sont soit le résultat d'une mauvaise utilisation, soit celui d'une intoxication volontaire. En milieu rural en particulier, les intoxications suicidaires ne sont plus exceptionnelles et leur nombre est souvent sous-estimé. En effet, faute de moyens, les intoxications aiguës par les pesticides ne sont pas toujours bien diagnostiquées ni bien documentées (1-3). De plus, les doses létales ne sont pas connues pour la plupart des pesticides. Dans la plupart des cas d'intoxication volontaire, l'ingestion de plusieurs pesticides ou xénobiotiques associés complique le diagnostic. Si les organophosphorés, les organochlorés et les ammoniums quaternaires sont à l'origine d'une majorité des intoxications, du fait de leur utilisation croissante les autres classes de pesticides (pyrethrinoïdes, carbamates) semblent de plus en plus impliquées dans les intoxications humaines. Paradoxalement, parmi les cas d'intoxication publiés, peu de données quantifiées et de données humaines sont disponibles dans la littérature $(1,4,5)$. Il semble donc nécessaire que le diagnostic d'intoxication aiguë soit complété par des méthodes analytiques quantitatives, spécifiques et sélectives $(6,7)$.

La plupart des méthodes de dosage chromatographiques de pesticides dans les matrices biologiques publiées ne permettent de doser qu'un faible nombre de pesticides. Parmi ces méthodes chromatographiques, la chromatographie en phase gazeuse (CPG) reste la plus utilisée pour les pesticides volatils et non thermosensibles (8-10). Ainsi, couplée avec des méthodes de détection classiques comme la détection par ionisation de flamme (FID) ou sélectives comme la détection azote-phosphore (NPD) ou la détection par capture d'électrons, la CPG permet de doser les organophosphorés, les organochlorés, les amides, les carbamates tory for all. Limits of detection (LODs) and limits of quantitation (LOQs) ranged respectively from 2.5 to $20 \mathrm{ng} / \mathrm{ml}$ and from 5 to $50 \mathrm{ng} / \mathrm{ml}$. An excellent linearity was observed from LOQs up to $1000 \mathrm{ng} / \mathrm{ml}$ for all the pesticides studied. The proposed procedures yielded reproducible results with good inter-assay accuracy and precision. A few cases of intoxication are presented to demonstrate the diagnostic interest of these methods: in two cases were determined lethal concentrations of endosulfan and carbofuran; in three other cases, the procedures helped diagnose intoxication with respectively parathion-ethyl, bifenthrin and aldicarb.

\section{KEY-WORDS}

Pesticides, GC/MS, LC/MS, SPE, serum, clinical toxicology; forensic toxicology.

ainsi que les pyréthrinoïdes. La chromatographie liquide (CLHP) est la méthode adaptée pour les molécules polaires ou thermosensibles (11-13), telles que les carbamates, les pyréthrinoïdes ou les triazines. Les méthodes de détection spectrométriques à barrette de diodes (UV-BD) ou fluorimétrique (FL) sont utilisées quotidiennement, mais ne sont pas suffisamment spécifiques ou universelles (14). La détection par spectrométrie de masse reste actuellement le seul mode de détection vraiment spécifique, et quasiment universel. Ainsi, des méthodes utilisant la spectrométrie de masse en tandem (GC-MS-MS, LC-MS-MS) s'avèrent performantes pour doser les pesticides et leurs métabolites dans l'urine ou le sang avec des sensibilités élevées, en éliminant les nombreuses interférences coextraites (14, 15). En particulier, les couplages LC-MS et LC-MSMS utilisant soit l'ionisation à pression atmosphérique par thermospray (TS), électrospray (ES) ou l'ionisation chimique à pression atmosphérique (APCI) prennent une part croissante dans les méthodes publiées, du fait de leur sensibilité et de leur facilité d'utilisation (16, 17). La présence, dans les pesticides, de métaux comme le fer, le manganèse et le zinc peut être détectée par spectrométrie d'absorption atomique (AAS) ou par ionisation par plasma induit-spectrométrie de masse (ICP-MS) (18).

L'extraction liquide/liquide (LLE) est encore largement utilisée dans les méthodes de screening de pesticides dans les matrices biologiques. Toutefois, l'extraction sur support solide (SPE) et la micro-extraction sur support solide (SPME) permettent d'éviter notamment la formation d'émulsions souvent observées en LLE. Par ailleurs, les propriétés physico-chimiques des supports polymériques ont largement contribué à leur essor, en améliorant la rétention et la purification des analytes, mais aussi en simplifiant les procédures d'extraction. Ainsi, la SPME développée par Pawliszyn $(19,20)$ permet de réaliser l'extraction dans les matrices biologiques, la concentration et l'injection en une seule 
étape. En toxicologie d'urgence, la SPME présente l'avantage d'être rapide, mais elle est peu sensible ; la LLE est peu onéreuse, cependant elle demande plus de temps de manipulation ; enfin la SPE a l'avantage d'être sensible et automatisable, mais elle est d'un coût plus élevé. Les méthodes publiées sont en général complexes $(21,22)$ : les méthodes d'extraction sont longues et une étape de dérivation est souvent nécessaire. Elles ne sont donc pas applicables dans des cas d'intoxications en urgence. Ainsi, il nous a semblé important de développer des méthodes simples, rapides et spécifiques, permettant l'identification et la quantification de nombreux pesticides dans les matrices biologiques par spectrométrie de masse. Des exemples de cas d'intoxication pour lesquels ces méthodes ont été utilisées sont décrits, afin de démontrer leur intérêt dans le diagnostic toxicologique.

\section{Matériel et méthode}

\section{Matériel}

Tous les solvants et réactifs proviennent de chez Prolabo (Fontenay-sous-Bois, France). L'ensemble des 61 pesticides ont été fournis par Cluzeau Info Labo (Sainte-Foy-La-Grande, France). Le tampon phosphate $(0,1 \mathrm{M})$ est préparé après dissolution de $6,81 \mathrm{~g}$ de phosphate de potassium monosodique dans $450 \mathrm{ml}$ d'eau désionisée, ajusté à pH 7,0 avec de l'hydroxyde de potassium $(1,0 \mathrm{M})$, jusqu'à un volume total de $500 \mathrm{ml}$. Une solution de méthanol alcalin est préparée en ajoutant $2,5 \mathrm{ml}$ d'ammoniaque $(3 \mathrm{~N}$ ) dans $47,5 \mathrm{ml}$ de méthanol.

\section{Couplage chromatographie en phase gazeuse/spectrométrie de masse (GC/MS)}

Le couplage GC/MS utilisé est un spectromètre de masse Shimadzu QP 5000 couplé à un chromatographe en phase gazeuse Shimadzu GC 17A, équipé d'un injecteur automatique AOC 14000 et du logiciel Class 5000 version 2.2 (Shimadzu, Courtaboeuf, France). La colonne capillaire est une Supelco PTE5 $5 \%$ phenyl-95\% méthyl siloxane ( $30 \mathrm{~m}$ x 0,25 mm d. i., 0,25 $\mu \mathrm{m}$ d'épaisseur de film). L'injecteur est utilisé dans le mode splitless et sa température est fixée à $250^{\circ} \mathrm{C}$. Le gaz vecteur est de l'hélium $\mathrm{N} 55$ et son débit est fixé à $2,1 \mathrm{ml} / \mathrm{min}$. Le même gradient de température est utilisé pour les 29 pesticides organophosphorés et les 18 pesticides organochlorés. La température du four passe de $60^{\circ} \mathrm{C}$ (pendant $2 \mathrm{~min}$ ) à $280^{\circ} \mathrm{C}$ (pendant $5 \mathrm{~min}$ ) en $10^{\circ} \mathrm{C} / \mathrm{min}$. L'ionisation est réalisée par impact électronique $(70 \mathrm{eV})$.
La quantification est réalisée en mode fragmentométrique ou SIM (single ion monitoring) en suivant l'ion le plus intense pour la quantification, et quand cela est possible, deux ions pour la confirmation. L'identification des produits est réalisée en déterminant le temps de rétention relatif (RRT) du pic chromatographique et l'abondance relative des ions de confirmation par rapport à l'ion de quantification (Tableaux 1 et 2).

\section{Couplage chromatographie en phase liquide/spectrométrie de masse (LC/MS)}

Le couplage LC/MS est composé de pompes Shimadzu LC 10AD, d'un injecteur automatique series 200 Perkin-Elmer (Courtaboeuf, France) et d'un spectromètre de masse PE-Biosystems API 100 piloté par un logiciel Masschrom (PE Biosystems, Courtaboeuf, France). La colonne chromatographique est une Nucléosil C18, $5 \mu \mathrm{m}(150 \times 1 \mathrm{~mm}$ d.i. $)$ de Polymer Laboratories (Marseille, France). L'élution est réalisée à un débit de $50 \mu \mathrm{l} / \mathrm{min}$ en utilisant un gradient de $30 \%$ à $80 \%$ d'acétonitrile (ACN) dans un tampon de formiate d'ammonium ( $2 \mathrm{mM}, \mathrm{pH} 3$ ). L'ionisation est assurée par une source Ionspray ${ }^{\oplus}$.

La quantification est réalisée par fragmentométrie en mode SIM en suivant l'ion pseudomoléculaire (le plus abondant) pour la quantification, et quand cela est possible, deux ions pour la confirmation. L'identification des produits est réalisé en déterminant le temps de rétention relatif (RRT) du pic chromatographique et l'abondance relative des ions de confirmation par rapport à l'ion de quantification (Tableau 3).

\section{Méthodes d'extraction}

\section{Avant la GC/MS}

Les 47 pesticides volatiles (organophosphorés, organochlorés, phtalimides, pyréthrinoïdes et uraciles) dosés par GC/MS sont extraits sur cartouche Oasis ${ }^{\otimes}$ Hydrophily and Lypophily Balanced Matrix (HLB) (Waters, Guyancourt, France). Les cartouches sont conditionnées avec $1 \mathrm{ml}$ de méthanol et $1 \mathrm{ml}$ d'eau désionisée, puis est déposé $1 \mathrm{ml}$ de sérum surchargé avec $2 \mu \mathrm{g}$ de cyproheptadine comme étalon interne (EI). La colonne est ensuite lavée avec $2 \mathrm{ml}$ d'eau désionisée et séchée sous vide. L'élution des analytes est réalisée avec $3 \mathrm{ml}$ d'acétate d'éthyle, puis l'éluat est évaporé à sec. L'extrait sec est dissout dans $100 \mu \mathrm{l}$ d'acétate d'éthyle et $2 \mu \mathrm{l}$ sont injectés dans le couplage GC/MS. 
Tableau I : Ions sélectionnés, limites de détection (LDD) et de quantification $(L D Q)$ et gamme de linéarité de la méthode GC/MS pour le dosage de 29 pesticides organophosphorés dans le sérum.

\begin{tabular}{|c|c|c|c|c|c|}
\hline \multirow[t]{2}{*}{ PESTICIDES } & \multicolumn{2}{|c|}{$\begin{array}{c}\text { Ions sélectionnés } \\
(\mathrm{m} / \mathrm{z})\end{array}$} & \multicolumn{2}{|c|}{$\begin{array}{l}\text { Limites } \\
(\mu \mathrm{g} / \mathrm{I})\end{array}$} & \multirow[t]{2}{*}{$\begin{array}{c}\text { Linéarité } \\
(\mu \mathrm{g} / \mathrm{l})\end{array}$} \\
\hline & Quant. $^{(a)}$ & Conf. ${ }^{(b)}$ & LDD & LDQ & \\
\hline Azinphos-éthyle & 132 & 160 & 5 & 10 & $10-1000$ \\
\hline Azinphos-méthyle & 104 & 132 & 5 & 10 & $10-1000$ \\
\hline Bromophos-méthyle & 331 & 329 & 5 & 10 & $10-1000$ \\
\hline Cadusaphos & 159 & 158 & 5 & 10 & $10-1000$ \\
\hline Chlorpyriphos-éthyle & 197 & 314 & 5 & 10 & $10-1000$ \\
\hline Chlorpyriphos-méthyle & 288 & 125 & 5 & 10 & $10-1000$ \\
\hline Coumaphos & 362 & 226,210 & 5 & 10 & $10-1000$ \\
\hline Dimethoate & 125 & 172,229 & 5 & 10 & $10-1000$ \\
\hline Ethion & 231 & 384,153 & 5 & 10 & $10-1000$ \\
\hline Ethoprophos & 158 & 242,200 & 5 & 10 & $10-1000$ \\
\hline Fénitrothion & 277 & 260,125 & 5 & 10 & $10-1000$ \\
\hline Fenthion & 278 & 169,153 & 5 & 10 & $10-1000$ \\
\hline Fonophos & 246 & 137,109 & 5 & 10 & $10-1000$ \\
\hline Isophenfos & 213 & 255,185 & 5 & 10 & $10-1000$ \\
\hline Malathion & 125 & 173,158 & 5 & 10 & $10-1000$ \\
\hline Métidathion & 145 & 125,85 & 5 & 10 & $10-1000$ \\
\hline Mévinphos & 127 & 164,192 & 5 & 10 & $10-1000$ \\
\hline Parathion-éthyle & 291 & 97,109 & 5 & 10 & $10-1000$ \\
\hline Parathion-méthyle & 263 & 125,109 & 5 & 10 & $10-1000$ \\
\hline Phénamiphos & 303 & 288,217 & 5 & 10 & $10-1000$ \\
\hline Phorate & 260 & 231,121 & 5 & 10 & $10-1000$ \\
\hline Phosalone & 182 & 367,184 & 5 & 10 & $10-1000$ \\
\hline Phosmet & 160 & 161,317 & 5 & 10 & $10-1000$ \\
\hline Pirimiphos-éthyle & 318 & 333,109 & 5 & 10 & $10-1000$ \\
\hline Pirimiphos-méthyle & 290 & 305,125 & 5 & 10 & $10-1000$ \\
\hline Pyraphos & 221 & 232,373 & 5 & 10 & $10-1000$ \\
\hline Quinalphos & 146 & 298,157 & 5 & 10 & $10-1000$ \\
\hline Terbuphos & 231 & 186,153 & 5 & 10 & $10-1000$ \\
\hline Vamidothion & 87 & 215,229 & 5 & 10 & $10-1000$ \\
\hline
\end{tabular}

(a) Quant. : ion de quantification

(b) Conf. : ion de confirmation

\section{Avant la LC/MS}

Les 14 molécules polaires (carbamates, benzimidazoles) analysées par LC/MS sont extraites en utilisant des colonnes Oasis $^{\otimes}$ Matrix Cation Exchange (MCX) (Waters, Guyancourt, France). Les cartouches sont conditionnées successivement avec $1 \mathrm{ml}$ de méthanol et $1 \mathrm{ml}$ de tampon phosphate $0,1 \mathrm{M}$ (à $\mathrm{pH} 7$ ), puis est déposé $1 \mathrm{ml}$ de sérum surchargé avec $1 \mu \mathrm{g}$ de parbendazole utilisé comme étalon interne (EI). La colonne est lavée avec $1 \mathrm{ml}$ d'eau désionisée. Une première élution est réalisée avec $1 \mathrm{ml}$ de méthanol. Après un second lavage à avec $1 \mathrm{ml}$ d'une solution d'HCl $0,1 \mathrm{~N}$, une deuxième élution est réalisée avec $1 \mathrm{ml}$ de méthanol et $1 \mathrm{ml}$ de méthanol ammoniaqué $(5 \%)$. Lès phases organiques sont assemblées et évaporées à sec. L'extrait sec est dissout dans $100 \mu$ de phase mobile et $2 \mu l$ sont injectés dans le système LC/MS.

\section{Résultats et discussions}

\section{Performance des méthodes}

Pour les deux méthodes, utilisant la GC/MS et la LC/MS les rendements d'extraction obtenus à une concentration et à forte concentration varient selon les pesticides et sont satisfaisants (de 70 à $99 \%$ ). Les limites de détection (LOD) et les limites de quantification (LOQ) sont comprises entre 2,5 et $50 \mathrm{ng} / \mathrm{ml}$ et entre 5 et $100 \mathrm{ng} / \mathrm{ml}$, respectivement (Tableaux 1-3).

Les deux méthodes sont répétables avec une bonne précision (coefficient de variation, CV $<19 \%$ ). La linéarité a été vérifiée des LDQ respectives jusqu'à 1000 $\mathrm{ng} / \mathrm{ml}$ pour tous les pesticides, avec des coefficients de corrélation (r) entre 0,997 et 0,999. Par ailleurs, ces méthodes ont été testées sur des échantillons réels dans des cas d'intoxication. 
Tableau II : Ions sélectionnés, limites de détection (LDD) et de quantification (LDQ) et gamme de linéarité de la méthode GC/MS pour le dosage de 12 organochlorés, d'un phtalimide, d'un uracile, de deux triazines et d'un pyréthrinoïde dans le sérum.

\begin{tabular}{|c|c|c|c|c|c|}
\hline \multirow[t]{2}{*}{ PESTICIDES } & \multicolumn{2}{|c|}{$\begin{array}{c}\text { Ions sélectionnés } \\
(\mathrm{m} / \mathbf{z})\end{array}$} & \multicolumn{2}{|c|}{$\begin{array}{c}\text { Limites } \\
(\mu g /)\end{array}$} & \multirow[t]{2}{*}{$\begin{array}{c}\text { Linéarité } \\
(\mu \mathrm{g} / \mathrm{l})\end{array}$} \\
\hline & Quant.(a) & Conf. (b) $^{-1}$ & LDD & LDQ & \\
\hline ORGANOCHLORES & & & & & \\
\hline Alachlore & 159 & 188,237 & 5 & 10 & $10-1000$ \\
\hline Aldrine & 263 & 293,265 & 5 & 10 & $10-1000$ \\
\hline $\mathrm{DDE}$ & 318 & 246,248 & 5 & 10 & $10-1000$ \\
\hline DDT & 235 & 237,165 & 5 & 10 & $10-1000$ \\
\hline Dieldrine & 277 & 263,279 & 5 & 10 & $10-1000$ \\
\hline Endosulfan $\alpha$ & 195 & 237,339 & 5 & 10 & $10-1000$ \\
\hline Endosulfan $\beta$ & 195 & 229,341 & 5 & 10 & $10-1000$ \\
\hline Endosulfan sulfate & 229 & 272,385 & 5 & 10 & $10-1000$ \\
\hline Heptachlore & 272 & 237,337 & 5 & 10 & $10-1000$ \\
\hline Hexachlorobenzène & 284 & 286,249 & 5 & 10 & $10-1000$ \\
\hline Lindane & 181 & 219,183 & 5 & 10 & $10-1000$ \\
\hline Métolachlore & 162 & 238,240 & 5 & 10 & $10-1000$ \\
\hline $\begin{array}{l}\text { PHTALIMIDE } \\
\text { Captane }\end{array}$ & 151 & 79,80 & 5 & 10 & $10-1000$ \\
\hline $\begin{array}{l}\text { URACILE } \\
\text { Bromacile }\end{array}$ & 207 & 205,231 & 5 & 10 & $10-1000$ \\
\hline TRIAZINE & & & & & \\
\hline Atrazine & 200 & 215,173 & 5 & 10 & $10-1000$ \\
\hline Simazine & 202 & 186,173 & 5 & 10 & $10-1000$ \\
\hline $\begin{array}{l}\text { PYRETHRINOIDES } \\
\text { Bifenthrine }\end{array}$ & 181 & 165,166 & 5 & 10 & $10-1000$ \\
\hline
\end{tabular}

(a) Quant. : ion de quantification

(b) Conf. : ion de confirmation

\section{Cas cliniques}

\section{Cas 1 : intoxication par le l'endosulfan}

Un homme de 39 ans, ayant connu des épisodes dépressifs, a ingéré volontairement une quantité inconnue de pesticides. L'examen clinique a montré un syndrome cholinergique, avec transpiration et myosis. Peu après son admission, le patient a présenté des crises convulsives tonico-cloniques et a reçu du diazépam et du phénobarbital. Les symptômes cholinergiques et les crises convulsives ont persisté. Une tachycardie et une fibrillation ventriculaire ont été observées. Une désaturation en oxygène durant les crises a nécessité une oxygénothérapie. En dépit de tentatives de réanimation, le patient est décédé quelques heures plus tard.

Dans ce cas, les résultats de l'analyse ont permis de détecter et de doser des concentrations d' $\alpha$ - et de $\beta$ endosulfan de 6,5 et $1,3 \mathrm{mg} / \mathrm{l}$, respectivement (Fig. 1).

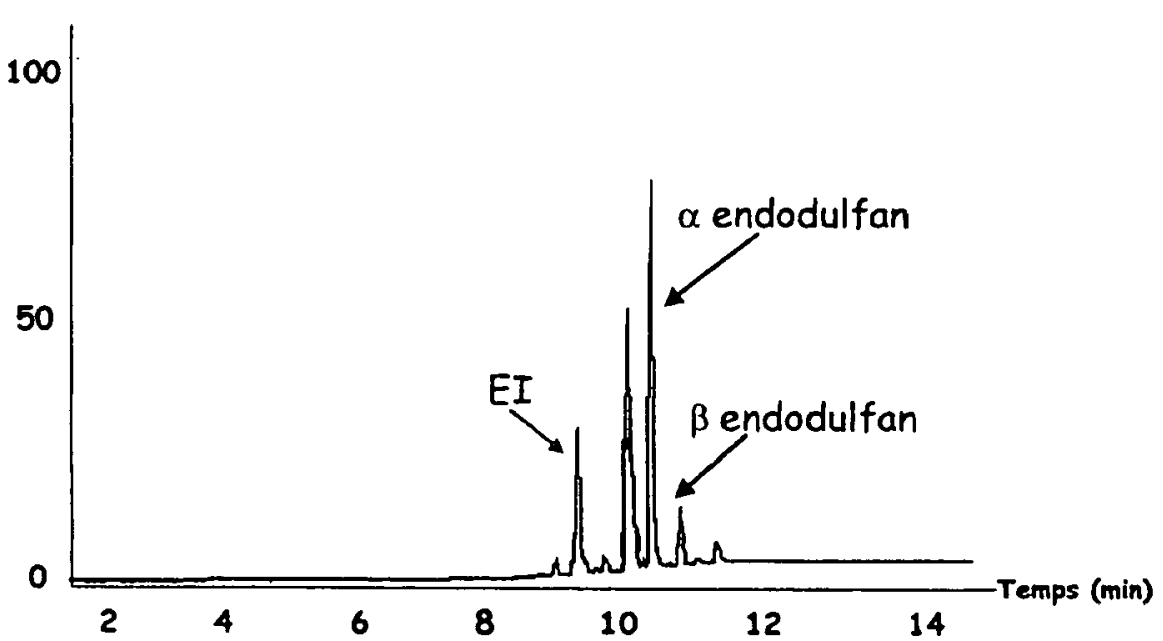

Figure 1 : Chromatogramme reconstitué de GC/MS d'un échantillon sérique de patient intoxiqué par l'endosulfan $(\alpha$-endosulfan 6,5 $\mathrm{mg} / \mathrm{l} ; \beta$-endosulfan $1,3 \mathrm{mg} / \mathrm{l})$. L'étalon interne utilisé était l'heptachlore au lieu de la cyproheptadine dans une première version de la méthode. 
Tableau III : Ions sélectionnés, limites de détection (LDD) et de quantification (LDQ) et gamme de linéarité de la méthode LC/MS pour le dosage de 11 carbamates et de 3 benzimidazoles dans le sérum.

\begin{tabular}{|c|c|c|c|c|c|}
\hline \multirow[t]{2}{*}{ PESTICIDES } & \multicolumn{2}{|c|}{$\begin{array}{c}\text { Ions sélectionnés } \\
(\mathbf{m} / \mathbf{z})\end{array}$} & \multicolumn{2}{|c|}{$\begin{array}{c}\text { Limites } \\
(\mu \mathrm{g} / \mathrm{/})\end{array}$} & \multirow[t]{2}{*}{$\begin{array}{c}\text { Linéarite } \\
(\boldsymbol{\mu} \mathrm{g} / \mathrm{l})\end{array}$} \\
\hline & Quant. ${ }^{(a)}$ & Conf. ${ }^{(\mathrm{b})}$ & LDD & LDQ & \\
\hline $\begin{array}{l}\text { CARBAMATE } \\
\text { Aldicarbe } \\
\text { Carbaryl } \\
\text { Carbofuran } \\
\text { Chlorprophame } \\
\text { Ethiofencarb } \\
\text { Fenoxycarb } \\
\text { Formétanate } \\
\text { Méthiocarbe } \\
\text { Méthomyl } \\
\text { Pirimicarbe } \\
\text { Prophame }\end{array}$ & $\begin{array}{c}89 \\
145 \\
123 \\
172 \\
107 \\
302 \\
110 \\
121 \\
88 \\
239 \\
138\end{array}$ & $\begin{array}{c}116 \\
155 \\
165 \\
126 \\
- \\
88 \\
93 \\
169 \\
106 \\
182 \\
120\end{array}$ & $\begin{array}{l}10 \\
20 \\
2,5 \\
50 \\
20 \\
10 \\
20 \\
50 \\
20 \\
2,5 \\
50\end{array}$ & $\begin{array}{c}20 \\
50 \\
5 \\
100 \\
50 \\
20 \\
50 \\
100 \\
50 \\
5 \\
100\end{array}$ & $\begin{array}{c}20-1000 \\
50-1000 \\
5-1000 \\
100-1000 \\
50-1000 \\
20-1000 \\
50-1000 \\
100-1000 \\
50-1000 \\
5-1000 \\
100-1000\end{array}$ \\
\hline $\begin{array}{l}\text { BENZIMIDAZOLE } \\
\text { Carbendazime } \\
\text { Thiabendazole } \\
\text { Thiophanate-méthyle }\end{array}$ & $\begin{array}{l}160 \\
202 \\
151\end{array}$ & $\begin{array}{l}192 \\
175 \\
343\end{array}$ & $\begin{array}{c}2,5 \\
2,5 \\
50\end{array}$ & $\begin{array}{c}5 \\
5 \\
100\end{array}$ & $\begin{array}{c}5-1000 \\
5-1000 \\
100-1000\end{array}$ \\
\hline
\end{tabular}

(a) Quant. : ion de quantification

(b) Conf. : ion de confirmation

Ces valeurs correspondent aux concentrations sanguines (23-25) de 4 et $30 \mathrm{mg} / \mathrm{l}$ rapportées par Coutselinis et al. (26) et Bernardelli et al. (23) dans des cas d'intoxication mortelle. Les symptômes d'empoisonnement aux organochlorés sont liés à la stimulation du système nerveux central avec hyperexcitabilité neuronale. Les principaux signes cliniques sont les convulsions, le coma, la dépression respiratoire pouvant conduire au décès (27-30). Le tableau s'accompagne d'une acidose métabolique, et peut se compliquer d'une rhabdomyolyse. Le diagnostic peut être confirmé et complété par un dosage sanguin du pesticide organochloré. Il existe en effet une assez bonne corrélation entre les effets cliniques et les concentrations (dans le cas du lindane par exemple).

\section{Cas 2 : intoxication par le carbofuran}

Un homme de 72 ans a été trouvé dans le coma avec un sachet de carbofuran à proximité. Un premier arrêt cardiaque a nécessité un massage, puis l'administration d'adrénaline. Le patient a été immédiatement intubé et ventilé mécaniquement. L'activité cardiaque s'est améliorée (pulsations $100 / \mathrm{min}$, pression sanguine $75 \mathrm{~mm}$ $\mathrm{Hg}$ ). Il a été admis à l'hôpital dans le coma (Glasgow 3). Son examen a montré une hypotension et des sécrétions bronchiques. Le tableau clinique s'est accompagné d'un myosis et d'une hypothermie $\left(31,2^{\circ} \mathrm{C}\right)$. Il a alors reçu de l'atropine. Une chute de l'activité cholinestérasique a été observée : activité de la cholinestérase globulaire de $0,42 \mathrm{U} / \mathrm{ml}$ et de la cholinestérase sérique de $1720 \mathrm{U} / \mathrm{l}$. Un traitement au pralidoxime à tout d'abord été administré, puis stoppé dès que l'intoxication aux carbamates a été diagnostiquée. Les autres anomalies biologiques étaient : une hyperkaliémie $(6,1 \mathrm{mmol} / \mathrm{l})$, une insuffisance rénale (créatininémie : $410,73 \mathrm{mmol} / \mathrm{l}$ ) et une cytolyse hépatique (SGPT : $1588 \mathrm{mU} / \mathrm{ml}$, SGOT : $1624 \mathrm{mU} / \mathrm{ml}$ ). Après une forte altération des fonctions respiratoires et cardiovasculaires, le patient est décédé $15 \mathrm{H}$ après son admission. Les concentrations de carbofuran mesurées ont été de $1,5 \mathrm{mg} / 1$ à $\mathrm{J} 1$ (Fig. 2) et de $1,3 \mathrm{mg} / 1$ à $\mathrm{J} 2$. La seule concentration sanguine létale publiée était de $9,7 \mathrm{mg} / 1$ (31). Les symptômes cliniques d'une intoxication aux carbamates sont comparables à ceux des organophosphorés $(3,30,32)$. En effet, ils provoquent une diminution de l'activité cholinestérasique, mais contrairement aux organophosphorés leur liaison avec les cholinestérases est réversible. Dans ce cas, le résultat de l'analyse toxicologique a permis d'arrêter le traitement par les oximes (en l'occurrence le pralidoxime), car il n'est pas recommandé comme antidote dans les cas d'intoxication aux carbamates. Il peut en effet aggraver la symptomatologie des intoxications au carbaryl, par exemple (5). 


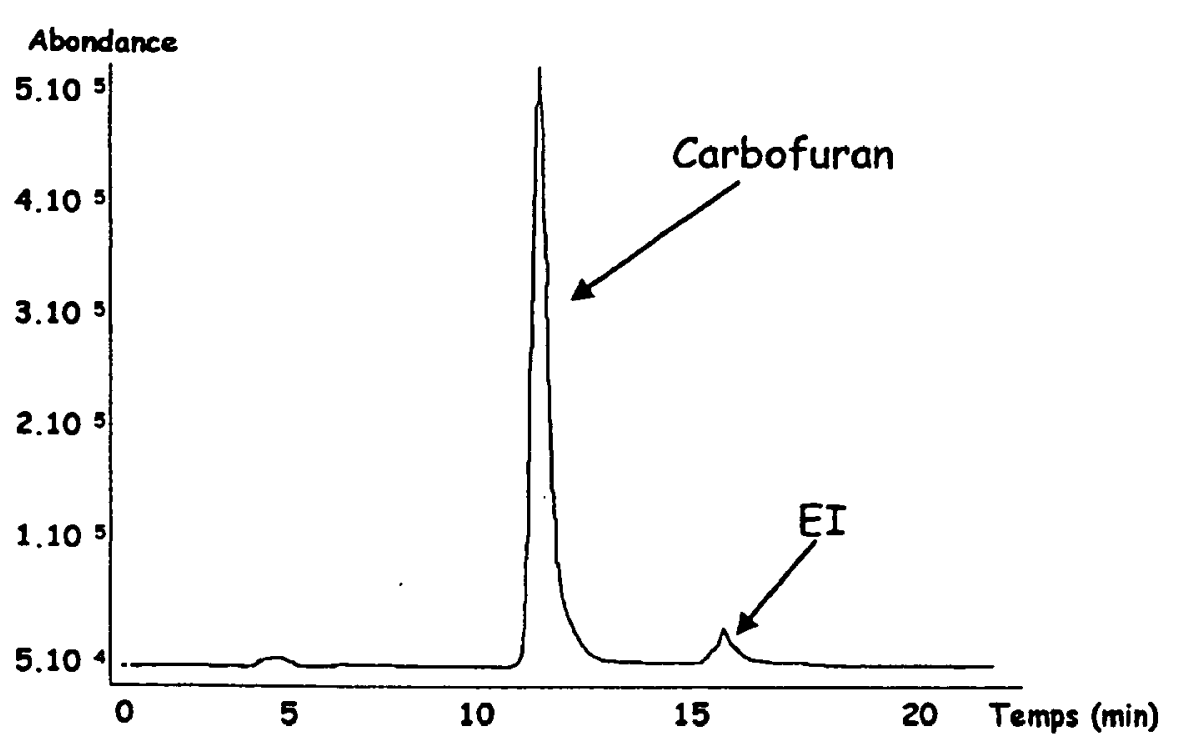

Figure 2 : Chromatogramme reconstitué de LC/MS d'un échantillon sérique de pateint intoxiqué par le carbofuran $(1,5 \mathrm{mg} / \mathrm{l})$.

\section{Cas 3 : intoxication par la bifenthrine}

Un homme de 80 ans a ingéré une solution insecticide contenant de la bifenthrine. Il a été admis en urgence à l'hôpital conscient, et les tests biologiques réalisés ont montré une hypoalbuminémie avec un taux de prothrombine à $62 \%$ et une faible hypoxémie. L'électrocardiogramme et les examens cardiaques et pulmonaires étaient normaux. La tomographie cérébrale s'est révélée normale elle aussi, exception faite de quelques calcifications bilatérales. L'évolution clinique a été favorable et le patient a pu quitter l'hôpital après quelques jours.

Les cas d'intoxication aiguës aux pyréthrinoïdes sont rares $(1,7)$. Les ingestions suicidaires se manifestent essentiellement par la stimulation du SNC et par des troubles musculaires (tremblements, myoclonies, convulsions) pouvant aboutir à la catatonie. Des manifestations ORL et respiratoires (toux, bronchospasme) peuvent être observées chez les sujets sensibles.

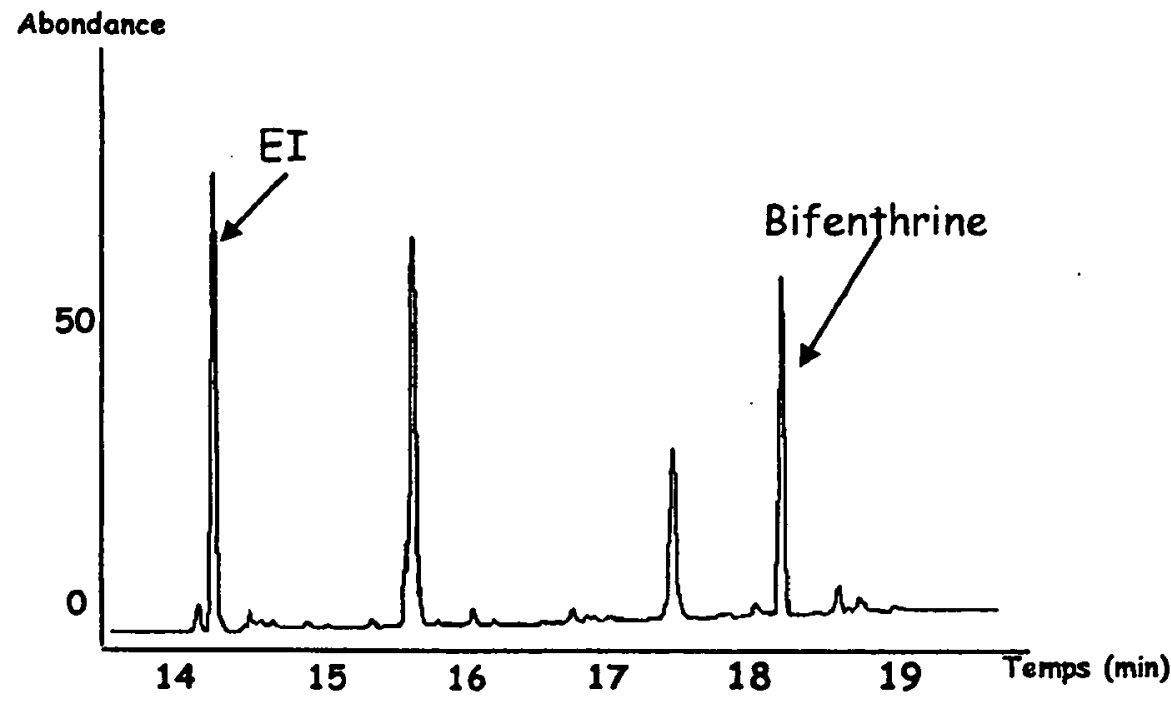

Figure 3 : Chromatogramme reconstitué de GC/MS d'un échantillon sérique de patient intoxiqué par la bifenthrine $(20 \mu \mathrm{g} / \mathrm{l})$. L'étalon interne utilisé était l'heptachlore au lieu de la cyproheptadine dans une première version de la méthode.
Généralement, le traitement est symptomatique. L'adrénaline est requise contre les réactions d'hypersensibilité aux pyréthrinoïdes. Il n'existe aucun antidote contre l'intoxication aux pyréthrinoïdes. Une concentration fatale à $710 \mathrm{mg} / \mathrm{l}$ a été rapportée suite à un contact sur la peau, en milieu professionnel (7). Notre méthode a permis de déterminer une concentration de $20 \mu \mathrm{g} / \mathrm{l}$ dans le sérum (Fig. 3), en accord avec la symptomatologie et l'évolution clinique du patient.

\section{Cas 4 : intoxication par le parathion- éthyle}

Un homme de 59 ans ayant connu plusieurs épisodes dépressifs, a ingéré volontairement une solution de Rhodiatox ${ }^{\circledast}$ contenant $10 \%$ de parathion-éthyle. Dès l'admission aux urgences, une dépression respiratoire accompagnée d'une mydriase, d'un strabisme et d'une vision trouble ont été observés. La mesure de l'activité cholinestérasique a montré une chute de l'activité globulaire $(0,8 \mathrm{UI} / \mathrm{ml}$, normale : 2,3-4,7 ; sérique $(1553$ $\mathrm{U} / 1$ à $\mathrm{J} 1,776 \mathrm{U} / 1$ à $\mathrm{J} 3,810 \mathrm{U} / 1$ à $\mathrm{J} 4$; normale : 700019000 U/l). Du pralidoxime ( $200 \mathrm{mg} / 24 \mathrm{~h}$ ) a ensuite été administré au patient. L'examen clinique a montré des fasciculations musculaires. Après un arrêt cardiaque, un massage a été réalisé et de l'adrénaline administrée au patient, qui a été ensuite intubé et ventilé. Le tableau s'est accompagné d'une tachycardie ventriculaire, d'une hypertension et d'un myosis. L'état général s'est peu à peu amélioré, et le patient a quitté l'hôpital 3 jours après son admission.

Les signes comme les troubles digestifs (nausées, douleurs abdominales), les hypersécrétions muqueuses, les signes neuromusculaires, l'altération de la vision, les fasciculations musculaires sont caractéristiques d'une intoxication par les organophosphorés. Les troubles métaboliques et cardiovasculaires représentent des complications classiques. Le traitement comprend des mesures de réanimation symptomatiques (intubation et ventilation assistée, ...). Le traitement spécifique fait appel à l'atropine qui est donnée pour préserver les fonctions vitales dans des cas d'intoxication sévère et à un antidote comme le pralidoxime qui doit être administré dès que possible (7).

Le parathion-éthyl est considéré comme très toxique parmi les organophosphorés [1, 13, 30, 33-34]. Il est fréquemment utilisé dans les suicides $(7,35)$. Ainsi, un cas d'intoxication mortel a été publié avec une concentration sérique de $70 \mu \mathrm{g} / \mathrm{l}$ (36). Dans le cas présent, notre méthode a permis de déterminer une concentration non létale de $213 \mu \mathrm{g} / \mathrm{l}$ (Fig. 4). 


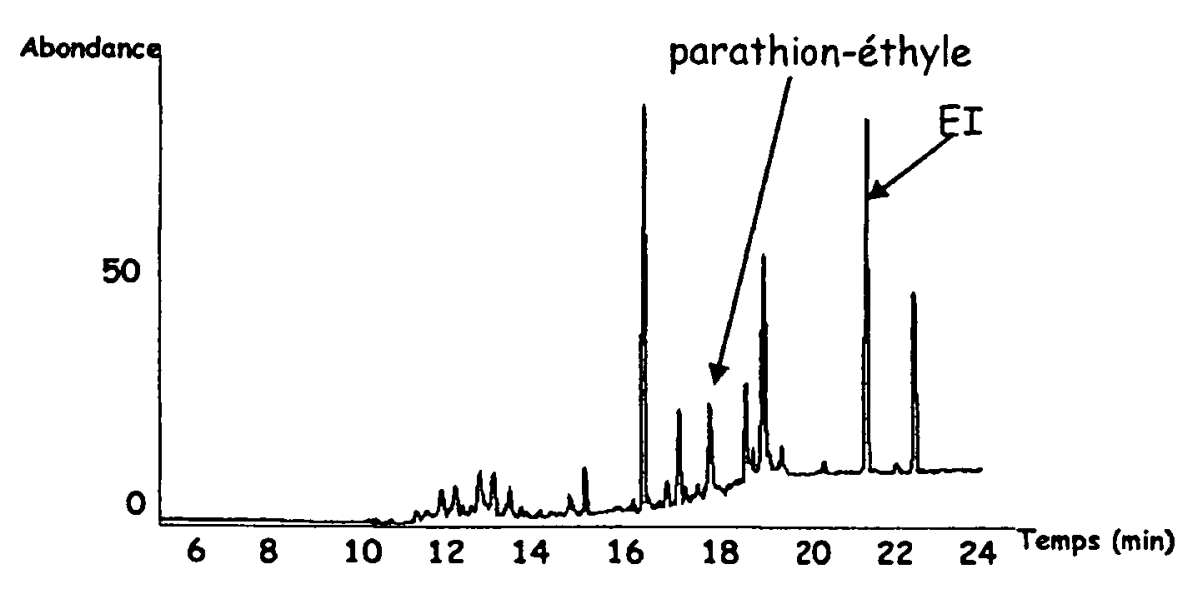

Figure 4 : Chromatogramme reconstitué de GC/MS d'un échantillon sérique de patient intoxiqué par le parathionéthyle $(213 \mu \mathrm{g} / \mathrm{l})$.

\section{Cas 5 : intoxication par l'aldicarbe}

Un homme de 57 ans, hypertendu, est transporté aux urgences dans le coma. Il présente des signes cholinergiques, avec un myosis mais aussi une hypersécrétion bronchique avec dyspnée et une hyperthermie $\left(33,2^{\circ} \mathrm{C}\right)$. Le patient est immédiatement intubé et ventilé. Devant ces symptômes muscariniques, une intoxication par les organophosphorés a été suspectée, puis confirmée dans un premier temps par une diminution de l'activité cholinestérasique. Du pralidoxime et de l'atropine ont donc été administrés comme antagonistes. Après extubation (à J3), l'évolution a été favorable.

La méthode analytique développée nous a permis d'identifier l'aldicarbe comme responsable de l'intoxication (Fig. 5), mais non de le quantifier car la matrice étudiée était du liquide gastrique en très faible quantité. L'aldicarbe est considéré comme fortement toxique comparé aux autres carbamates ; la concentration sanguine non fatale la plus élevée publiée est de $3 \mathrm{mg} / \mathrm{l}$ (37-39).

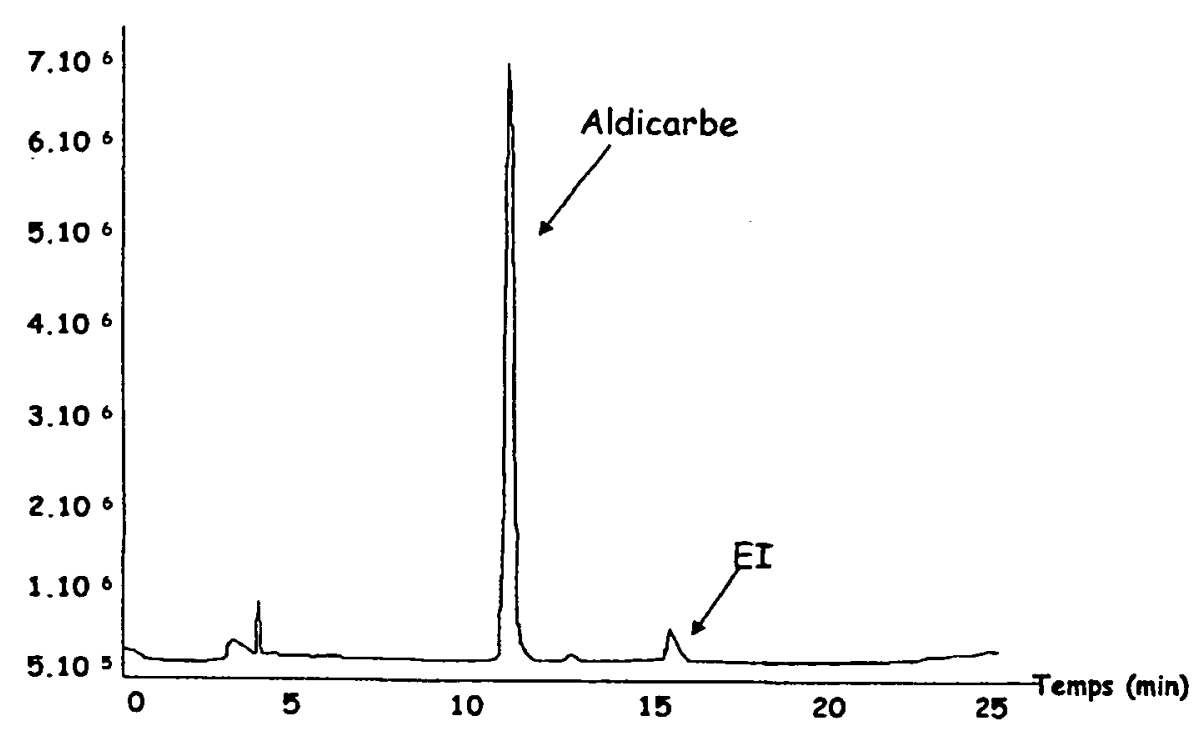

Figure 5 : Chromatogramme reconstitué de LC/MS d'un échantillon sérique de patient intoxiqué par l'aldicarbe.

\section{Discussions}

Les méthodes analytiques présentées ici utilisent l'extraction sur phase solide (SPE) et les couplages GC/MS et LC/MS, permettant l'identification et le dosage de 61 pesticides (organophosphorés, organochlorés, phtalimides, uraciles, pyréthrinoïdes, carbamates et benzimidazoles), de volatilité, de polarité et de stabilité thermiques variables, et souvent impliqués dans des intoxications aiguës (Tableaux 1-3). Les cartouches d'extraction SPE munies de supports polymériques tels que OASIS $^{\oplus}$ HLB et MCX $(40,41)$, favorisent la rétention de molécules aussi bien polaires qu'apolaires et diminuent l'influence du $\mathrm{pH}$ sur la rétention. Par ailleurs, l'utilisation de couplages LC/MS et GC/MS confère à cette méthode une haute spécificité et l'acquisition en mode fragmentométrique une sensibilité élevée (42). Les carbamates (aldicarbe et carbofuran) sont dosés par LC/MS, alors que les organophosphorés (parathionéthyl), les organochlorés (endosulfan), les uraciles (bromacile) et les pyréthrinoïdes (bifenthrine) sont dosés avec une plus grande sensibilité à l'aide d'un couplage GC/MS. Pour des questions de résolution chromatographique, il a été nécessaire de créer deux méthodes GC/MS différentes (bien qu'elles partagent les mêmes conditions chromatographiques), l'une pour 29 organophosphorés (tableau 1) et l'autres pour 18 pesticides (tableau 2). Les limites de quantification obtenues sont parmi les plus faibles publiées à ce jour $(9,16,17,24,43)$. De plus, les méthodes utilisées sont simples, sans étapes de lavage supplémentaire ni dérivation. Dans les cinq cas cliniques étudiés, l'identification et la quantification du pesticide en cause ont aidé au diagnostic final. En effet, la certitude diagnostique est parfois limitée par la nature ou la quantité de matrices biologiques disponibles ou par la performance de la méthode analytique utilisée (44).

Généralement, le traitement des intoxications par les pesticides est symptomatique, exception faite des organophosphorés pour lesquels les oximes sont des antidotes spécifiques. Au contraire, après une intoxication par les carbamates l'administration de pralidoxime $\left(\right.$ Contrathion ${ }^{\circledast}$ ) comme antidote, est considérée comme inutile et peut même aggraver la symptomatologie. En effet, les oximes modifient les structures des cholinestérases, alors que la liaison carbamate/enzyme n'est pas covalente, contrairement à celles des organophosphorés $(1,38)$. Ainsi, le traitement par les oximes a été arrêté dans deux des cas cliniques rapportés, dès que l'intoxication par les carbamates a été connue. Devant l'observation de symptômes muscariniques et d'une diminution de l'activité cholinestérasique, il est donc intéressant de pouvoir différencier une intoxication par les organophosphorés d'une intoxication par les carba- 
mates. Toutefois, les poly-intoxications peuvent provoquer de nombreux symptômes cliniques qui ne sont pas toujours caractéristiques. L'analyse toxicologique peut ainsi être déterminante pour le traitement des intoxications aiguës, aussi bien que pour l'investigation des cas médico-légaux.

\section{Conclusion}

Nous avons développé des méthodes sensibles, spécifiques et complémentaires pour l'identification et le dosage de pesticides appartenant à différentes classes. Elles sont simples et adaptées au diagnostic rapide d'intoxication aiguë, et peuvent aussi bien être utilisées pour documenter des cas médico-légaux.

\section{Références}

1. F.P. Kaloyanova, M.A. El Batawi, Human Toxicology of Pesticides, CRC Press, London, 1991 : 3-157.

2. S. Watanabee, detection of organophosphate pesticides in blood serum from the patients suspected of acute and chronic pesticides poisonings and its clinical significance 1972, $107: 301-302$.

3. M. Lifshitz, E. Shahak, A. Bolotin, S. Sofer, Carbamate and organophosphate poisoning in young children, pediatr. Emerg. Care 1999, 15 (2) : 102-103.

4. T.A. Gossel, J.D. Bricker, Principles of Clinical Toxicology, 3 rd Ed., Raven Press, NY, pesticides 1994 : 147-178.

5. F. Testud, Eds ESKA, Pathologie toxique en milieu de travail, 1998, chap 25, 341-363.

6. D.B. Barr, J.R. Barr, W.J. Driskell, R.H. Hill, D.L. Ashley, L.L. Needham, S.L. Head, E.J. Sampson, Strategies for biological monitoring of exposure for contemporary-use pesticides, Toxicological and Industrial Health $1999 ; 15$ (1-2) : 168-179.

7. He Fengsheng, Biological monitoring of occupational pesticides exposure, Int. Arch. Occup. Environ. Health $1993 ; 65: 569-576$.

8. T. Kojima, M. Yashiki, M. Ohtani, F. Chikasue, T. Miyazaki, Determination of dimethoate in blood and hemoperfusion cartridge following ingestion of formothion: a case study, Forensic Science International $1990 ; 48: 79-88$.

9. F. Musshoff, H. Junker, B. Madea, Rapid analysis of parathion in biological samples using headspace solidphase micro-extraction (HS-SPME) and gas chromatography/mass spectrometry (GC/MS), Clin. Chem. Lab. Med. $1999 ; 37$ (6) : 639-642.

10. Z. Vasilic, S. Bozena, V. Drevenkar, Dimethylphosphorus metabolites in serum and urine of persons poisoned by malathion or thiometon, ChemicoBiological Interactions $1999 ; 119$ : 479-487.

11. J. Demeter, A. Heyndrickx, Two lethal Endosulfan Poisonings in man, J. Anal. Toxicol. 1978 ; 2 : 68-74.

12. V.K. Sharma, R.K. Jadhav, G.J. Rao, A.K. Saraf, H. Chandra, High performance liquid chromatographic method for the analysis of organophosphorus and carbamates pesticides, Forensic Science International 1990 ; $48: 21-25$.
13. K. Futagami, C. Narazaki, Y. Kataoka, H. Shuto, R. Oishi, Application of high-performance thin-layer chromatography for the detection of organophosphorus insecticides in human serum after acute poisoning, $\mathrm{J}$. Chromatogr. B Biomed Sci Appl. 1997 ; 704 : 369-373.

14. T. Kumazawa, O. Suzuki, Separation methods for amino group-possessing pesticides in biological samples, J. Chromatogr. B $2000 ; 747$ : 241-254.

15. W.J. Driskell, R.H. Hill, D.B. Shealy, R .D. Hull, C.J. Hines, Identification of a major human urinary metabolite of alachlor by LC-MS/MS, Bull. Environ. Contam. Toxicol. 1996 ; 56 (6) : 853-859.

16. S. Kawasaki, H. Ueda, H. Itoh, J. Tadano, Screening of organophosphorus pesticides using liquid-chromatography/mass spectrometry, J. Chromatogr. $1992 ; 595$ 2) : 193-202.

17. H. Itoh, S. Kawasaki, J. Tadano, Application of liquidchromatography/mass spectrometry to pesticide analysis, J. Chromatogr. A 1996 ; $754:$ 61-76.

18. M. Marroni, C. Colosio, A. Ferioli, A. Fait, Biological monitoring of pesticide exposure : a review, Toxicology $2000 ; 143: 5-123$.

19. H. Lord, J. Pawliszyn, Evolution of solid-phase microextraction technology, J. Chromatogr. $2000 ; 885$ (1-2) : 153-193.

20. J. Pawliszyn, Solid Phase Microextraction- Theory and Practice, Ed. Wiley-VCH, 1997 New York.

21. S.J. Reid, R.R. Watts, A method for the determination of dialkyl phosphates residues in urine, J. Anal. Toxicol. $1981 ; 5$ (3) : 126-132.

22. A. Tuonaimen, J. Kangas, J. Liesivuori, A. Manninem, Biological monitoring of deltametrin in greenhouses, INT. Arch. Occup ; Environ. Health 1996 ; 69 : 62-64.

23. B. C. Bernardelli, M. C. Gennari, Death caused by ingestion of endosulfan, J. For. Sc., JFSCA 1987, 32 (4) : 1109-1112.

24. S.M. Waliiszewski, G.A. Szymczynski, Persistent Organochlorine Pesticides in blood serum and whole blood, Bull. Environ. Contam. Toxicol. 1991; 46 : 803809.

25. W.G. Grimmett, I. Dzendolet, I. Whyte, Intravenous thiodan, Clinical Toxicology $1996 ; 34$ (4) : 447-452. 
26. A. Coutselinis, P. Kentarchou, D. Boukis, Concentration levels of endosulfan in biological material (report of three cases), Forensic Science 1978 ; 11 : 75-76.

27. J.L. Blanco-Coronado, M. Repetto, R.J. Ginestal, J.R. Vicente, F. Yelamos, A. Lardelli, Acute intoxication by endosulfan, Clinical toxicology $1992 ; 30$ (4) : 575-583.

28. A.K. Sood, S.P. Yadav, S. Sood, Endosulphan poisoning presenting as status epileptyicus, Indian J. Med. Sci. 1994 ; 48 : 68-69.

29. R. Garnier, Acute tubular necrosis following endosulfan insecticide poisoning, Clinical toxicology $1995 ; 33$ (4) : 375-378.

30. R. Garcia-Repetto, M.L. Soria, M.P. Gimenez, M. Menendez, M. Repetto, Deaths from pesticides poisoning in Spain from 1991 to 1996, Vet. Hum. Toxicol. 1998, 40(3) : 166-168.

31. K. Sancewicz-Pach, B. Groszek, D. Pach, M. Klys, Acute pesticides poisonings in pregnant women, Przegl Lek $541997 ; 10: 741-744$.

32. Y. Iwata, J.B. Knaak, M.E. Dusch, J.R. O'Neal, J.L. Pappas, Worker re-entry research for carbofuran applied to California citrus tress, J. Agric. Food Chem. 1983; 31 (6) : 1131-1136.

33. R.H. Dreisbach, W.O. Robertson, Hanbook of poisoning, Los Atlos, Appleton and Lange publications, 1982.

34. J. De Bleecker, K. Van den Neucker, K. Colardyn, Intermediate syndrome with delayed distal polyneuropathy from ethyl parathion poisoning, Vet Hum. Toxicol. $1998 ; 40(6): 349-352$.

35. A. Ohberg, J. Lonquist, S. Sarna, E. Vuori, A. Penttila, Trends and avaibility of suicide methods in Finland, British J. Of Psychiatry 1995 ; $166: 35-43$.
36. J. Hamen, R. Wennig, Diagnostis of acute parathion intoxication and medical-legal consequences, Acta Clin. Belg. Suppl. $1999 ; 1$ : 54-58.

37. J.L. Burgess, J.N. Bersnstein, K. Hurlbut, Aldicarb poisoning. A case report with prolonged cholinesterase inhibition and improvement after pralidoxime therapy, Arch. Interm. Med. $1994 ; 154$ (2) : 221-224.

38. M. Lifshitz, M. Rotenberg, S. Sofer, T. Tamari, E. Shahak, S. Almog, Carbamates poisoning and oxime treatment in children: a clinical and laboratory study, Pediatrics 1994 ; 93(4) : 652-655.

39. A. Covaci, P. Manirakiza, V. Coucke, R. Beckers, P.G. Jorens, P. Schepens. A case of aldicarb poisoning : a possible murder attempt, J. Anal. Toxicol. $1999 ; 23$ (4) : 290-293.

40. X.-H. Chen, J.-P. Franke, J. Wijsbeek, R.A. de Zeeuw, Isolation of acidic, neutral and basic drugs from whole blood using a single mixed-mode solid-phase extraction column, J. Anal. Toxicol. 1992 ; 16 : 351-355.

41. M. Perruzi, G. Bartolucci, F. Cioni, Determination of phenoxyalkanoic acids and other herbicides at the $\mathrm{ng} / \mathrm{ml}$ level in water by solid-phase extraction with poly(divinylbenzene-co- $\mathrm{N}$-vinylpyrrolidone) sorbent and highperformance liquid chromatography-diode-array detection, J. Chromatogr. A $2000 ; 867: 169-175$.

42. J. Yinon, Forensic mass spectrometry, CRC Press, USA, Florida 1991 : 74-79.

43. G. Mariani, E. Benfenati, R. Fanelli, a NICI-GC-MS method to analyse endosulfan in biological samples, Inter. J. Environ. Anal. Chem. 1995 ; 58 : 67-72.

44. Y. Yang, T.C. Tsao, J.L. Lin, R.K. Lyu, P.C. Chiang, Carbofuran induced delayed neuropathy, J. Toxicol. Clin. Toxicol. $2000 ; 38$ (1) : 43-46. 